\title{
Avaliação do Perfil Sociodemográfico, Clínico-Laboratorial e Terapêutico dos Pacientes com Artrite Reumatóide que Participaram de Projetos de Pesquisa na Escola Paulista de Medicina, nos últimos 25 anos
}

\section{Evaluation of the Sociodemographic, Clinical-Laboratorial and Therapeutic Profile of Rheumatoid Arthritis Patients who Participated of Research Projects in the Escola Paulista de Medicina in the Last 25 Years}

\author{
Mirhelen Mendes de $\mathrm{Abreu}^{(1)}$, Sérgio Cândido Kowalski ${ }^{(2)}$, Rozana Mesquita Ciconelli ${ }^{(3)}$, \\ Marcos Bosi Ferraz ${ }^{(4)}$
}

\section{RESUMO}

Objetivo: descrever o perfil sociodemográfico, clínico-laboratorial e terapêutico dos pacientes com artrite reumatóide (AR) do ambulatório de reumatologia da Escola Paulista de Medicina (EPM-UNIFESP) que participaram de teses de pós-graduação no período de 25 anos, compreendido entre 1979 e 2004 . Pacientes e Métodos: teses que avaliaram pacientes com AR da EPM. Exclusão: teses que incluíram pacientes de outras instituições ou com outras doencas. Classificação dos estudos: conforme o período de conclusão - I (1979-1984); II (1985-1989); III (1990-1994); IV (1995-1999); V (2000-2004) - e tipo de estudo (pesquisa básica; reabilitação; radiologia; qualidade de vida; epidemiologia e economia de saúde). Resultados: Foram selecionadas 26 de 65 teses. Houve um predomínio de pesquisa básica ( 7 teses) nos três primeiros períodos, seguidos por reabilitação ( 6 teses) e de qualidade de vida ( 5 teses). Demografia: média de idade de 52 anos, predomínio do sexo feminino e raça branca. A escolaridade, avaliada em 5 estudos (qualidade de vida e economia de saúde), apresentou nível médio e superior de ensino nos últimos períodos. A classificação funcional (CF) foi pesquisada em 11 estudos, com maior prevalência da CF II. Tratamento: predomínio do uso de antiinflamatórios não-esteroidais (AINEs) e corticóides nos períodos I a III e, nos períodos III e $\mathrm{V}$, de drogas modificadoras de atividade de doença (DMARDs), especialmente de metotrexato (MTX). Conclusões: observou-se alteração no nível de escolaridade e predomínio dos estudos de pesquisa básica, reabilitação e qualidade de vida. Houve preferência inicial pelos AINEs e, atualmente, pelos DMARDs.

Palavras-chave: artrite reumatóide, história da medicina, tratamento, diagnóstico.

\begin{abstract}
Objective: To analyse the clinical, laboratory, treatment and social progress of patients with rheumatoid arthritis $(R A)$ who attend at Escola Paulista de Medicina (EPM-UNIFESP), Brazil, submitted to researching theses from 1979 to September, 2004. Patients and methods: 65 researches theses were reviewed and 26 were selected according inclusion/exclusion criterias. Inclusion: researches which analysed outpatients with $R A$ belonging to EPM ambulatory. Exclusion: researches which included outpatients from other institutions or diseases. Classifications of the studies: a) period of conclusion: I (1979-1984); II (1985-1989); III (1990-1994); IV (1995-1999); V(2000-2004); b) type of the study: laboratory; rehabilitation; radiology; epidemiology; quality of life; health economics. Results: Twenty six thesis out of 65 were suitable for analysis there was a predominance of basic research ( 7 thesis) on the first three periods, followed by reabilitation research ( 6 thesis) and measurement of quality of life ( 5 thesis). Concerning demographic data, patients were in average, 52 years old with prevalence of white females. High levels of schooling were noted for the last periods analysed. Patients functinal class were evaluated in 11 studies, with a predominance of functinal class II. Therapeutic strategies for the treatment of $R A$ have changed significantly, showing DMARDs in the therapeutic options. Conclusions: The demographic, clinical, laboratory, and therapeutic characteristics changed along time and type of this study developed. Initially there was a preference for NSAIDs, and nowadays for DMARDs.
\end{abstract}

Keywords: Rheumatoid arthritis, history of medicine, treatment, diagnosis.

\footnotetext{
Escola Paulista de Medicina (EPM), Universidade Federal de São Paulo (UNIFESP). Recebido em 20/10/05. Aprovado, após revisão, em 09/03/06.

1. Pós-graduanda em Reumatologia (EPM-UNIFESP).

2. Médico Voluntário da Disciplina de Reumatologia (EPM-UNIFESP).

3. Professora afiliada da Disciplina de Reumatologia (EPM-UNIFESP), Membro do Projeto Pronuclear da Sociedade Brasileira de Reumatologia (SBR)

4. Professor adjunto da Disciplina de Reumatologia (EPM-UNIFESP).

Endereço para correspondência: Mirhelen Mendes de Abreu, Rua Juréia, 372/85, Vila Mariana, São Paulo, CEP 04140-1 10, SP, Brasil, telefone/fax: 5579-6665, e-mail: mirhelen@terra.com.br
} 


\section{INTRODUÇÃO}

A artrite reumatóide (AR), paradigma das artropatias inflamatórias, pode ser considerada uma patologia recente, de origem no Novo Mundo ${ }^{(1,2)}$. De natureza crônica e incapacitante, afeta em torno de $1 \%$ da população mundial, o que a torna um sério problema de saúde pública, com graves conseqüências clínicas, psíquicas, sociais e econômicas ${ }^{(3,4)}$. Ao longo de décadas, várias medidas terapêuticas foram adotadas, visando minimizar os sintomas e, recentemente, busca-se também reduzir incapacidades e modular a evolução da doençca ${ }^{(5,6)}$.

Os progressos nas pesquisas clínica, farmacológica e laboratorial em AR e a inserção de novos parâmetros de avaliação da doença impactaram sua história natural, refletindo, também, o amplo arsenal medicamentoso e a postura mais agressiva em seu manejo ${ }^{(7)}$.

Por esses aspectos e por não haver estudo prévio que faça uma análise crítica das teses realizadas, o presente trabalho tem por objetivo descrever a população de pacientes com AR que participou de projetos de pesquisa de pós-graduação (mestrado e doutorado) na Escola Paulista de Medicina (EPM)/Universidade Federal de São Paulo (UNIFESP), e analisar as tendências metodológicas dos estudos realizados ao longo de 25 anos.

\section{PACIENTES E MÉTODOS}

Foram analisadas 65 teses sobre AR e selecionadas 26, conforme os critérios de seleção estabelecidos (Apêndice I). Critérios de inclusão: teses que avaliaram pacientes com AR acompanhados no ambulatório de reumatologia da Escola Paulista de Medicina (EPM)/ Universidade Federal de São Paulo (UNIFESP). Critérios de exclusão: teses que incluíram pacientes de outras instituições; teses que não avaliaram diretamente pacientes em seus estudos.

As características das 39/65 teses excluídas foram: 12 (30,7\%) que incluíram pacientes de outra instituição, 2 $(5,1 \%)$ que avaliaram apenas pacientes de outra instituição; $9(23,3 \%)$ que incluíram outras patologias na sua análise e não discriminaram a população por patologia; $5(12,8 \%)$ que não avaliaram pacientes ( 2 revisões sistemáticas e 3 de estudo experimental com animais); 7 (17,9\%) que avaliaram apenas pacientes com AR juvenil (ARJ) e 4 (10,2\%) que incluíram tanto pacientes com ARJ como AR em suas amostras.

As 26 teses selecionadas foram classificadas conforme dois critérios: quanto ao período de conclusão e o tipo de estudo abordado. Quanto ao período de conclusão, foram categorizados da seguinte forma: período I, 1979 - 1984; período II, 1985 - 1989; período III, 1990 - 1994; período IV, 1995-1999 e período V, 2000-2004.

De acordo com o padrão 'tipo de estudo', as teses foram subdivididas em: a) pesquisa básica (estudos executados em laboratório); b) estudos epidemiológicos, compreendendo os estudos que avaliaram alguma intervenção ou alteração clínica num determinado período de tempo; c) radiológico (estudos focando métodos de imagem); d) qualidade de vida (estudos com caráter de pesquisa em qualidade de vida); e) reabilitação e f) economia de saúde.

Foram analisados os critérios de seleção da amostra pelos estudos no decorrer de 25 anos. Os parâmetros demográficos incluíram sexo, média de idade, raça e nível de escolaridade. O perfil clínico-laboratorial analisado englobou parâmetros tais como: tempo de diagnóstico, compreendendo o período entre o início dos primeiros sintomas e o diagnóstico de AR; forma de apresentação; co-morbidades; classificação funcional; Health Assessment Questionnaire (HAQ); velocidade de hemossedimentação (VHS) e tratamento no momento do estudo. Neste último item, foram discriminadas as drogas utilizadas durante cada período de estudo analisado ${ }^{(8,9)}$.

A análise estatística utilizou medidas de tendência central e dispersão, visto que trata-se de um estudo puramente descritivo, não havendo, a priori, teste de hipóteses. Neste sentido, o estudo descreve, também, o critério de classificação para o diagnóstico de $\mathrm{AR}$ vigente e internacionalmente aceito na ocasião de cada trabalho, não sendo adotado, para a seleção deste trabalho, nenhum critério específico para o propósito da análise.

\section{RESULTADOS}

As teses analisadas foram desenvolvidas entre 1979 e 2004. Observou-se uma homogeneidade quanto aos critérios de inclusão dos estudos analisados, todos selecionando pacientes adultos acompanhados no ambulatório de reumatologia. A mudança observada nesse parâmetro se deu quanto aos critérios para o diagnóstico de AR. Para os estudos realizados até 1988 , utilizou-se o critério de classificação do American Rheumatism Association de $1958^{(10)}$. A partir deste período, utilizou-se o critério estabelecido pelo American College of Rheumatology (ACR), publicado em $1988^{(11)}$. Os critérios de exclusão também variaram conforme o período e o tipo de estudo avaliado. Das 26 teses analisadas, 11 (42,3\%) excluíram pacientes que apresentavam co-morbidades não relacionadas com 
AR. Dos 6 estudos sobre reabilitação avaliados, 2 excluíram pacientes com Classificação Funcional (CF) I e IV.

A Tabela 1 descreve a distribuição e classificação das teses ao longo de 25 anos, conforme o período de conclusão e o tipo de estudo avaliado. Nota-se uma variação nos tipos de estudo, com um predomínio de temas sobre pesquisa básica ( 7 teses; $26,9 \%$ ) nos três primeiros grupos (1979 -1984; 1985 - 1989 e 1990 - 1994). Nos grupos IV (1995 - 1999) e V (2000 - 2004), observa-se um crescente predomínio de temas relacionados à reabilitação $(6$ teses; $23 \%$ ), seguido por avaliação da qualidade de vida ( 5 teses; $19,2 \%)$. Em 2004, nota-se um incipiente surgimento de teses relacionadas à economia de saúde (2 teses; 7,6\%).

TABELA 1

DisTRIBUIÇÃO E CLASSIFICAÇÃO DAS TESES CONFORME O PERÍODO DE CONCLUSÃO E O TIPO DE ESTUDO

\begin{tabular}{|c|c|c|}
\hline $\begin{array}{c}\text { Período de conclusão } \\
\text { da tese }\end{array}$ & $\begin{array}{l}\text { Tipo de estudo } \\
\text { (n) }\end{array}$ & Total (26) \\
\hline Período I (1979-1984) & pesquisa básica (2) & 2 \\
\hline Período II (1985-1989) & pesquisa básica (2) & 2 \\
\hline Período III (1990-1994) & $\begin{array}{l}\text { qualidade de vida (2); } \\
\text { pesquisa básica (2); } \\
\text { epidemiologia (1) }\end{array}$ & 5 \\
\hline Período IV (1995-1999) & $\begin{array}{l}\text { qualidade de vida (3); } \\
\text { pesquisa básica (1); } \\
\text { reabilitação (3) }\end{array}$ & 8 \\
\hline Período V (2000-2004) & $\begin{array}{l}\text { qualidade de vida (2); } \\
\text { pesquisa básica (1); } \\
\text { reabilitação (3); } \\
\text { economia da saúde }(2) ; \\
\text { radiologia (1) }\end{array}$ & 9 \\
\hline
\end{tabular}

O total de pacientes incluídos neste trabalho foi 1424 (média: 54 ), dos quais, $92 \%$ pertenciam ao sexo feminino, com média de idade de 52 anos. É provável que alguns pacientes tenham participado de mais de um estudo ao longo do tempo avaliado (1979-2004). A variável raça foi analisada em 11 estudos, sendo incluídos 144,4 pacientes, em média. Destes, $71 \%$ eram pertencentes à raça branca (Tabela 2). A descrição do perfil socioeconômico foi caracterizada pela avaliação do estado civil, situação ocupacional e escolaridade. Apenas duas teses avaliaram situação ocupacional. Destas, $100 \%$ pertenciam ao grupo "economia da
TABELA 2

ANÁlise DAS TESES QUANTO A MÉDIA DE IDADE E SEXO DOS PACIENTES INCLUÍDOS NO ESTUDO

\begin{tabular}{|c|c|c|c|c|}
\hline \multirow{2}{*}{$\begin{array}{c}\text { Teses } \\
\text { analisadas }\end{array}$} & \multirow{2}{*}{$\begin{array}{l}\text { Total de pacientes } \\
\text { no estudo }\end{array}$} & \multirow{2}{*}{$\begin{array}{l}\text { Média de } \\
\text { idade (DP) }\end{array}$} & \multicolumn{2}{|c|}{ Sexo (F) } \\
\hline & & & $\mathrm{n}$ & $\%$ \\
\hline 1 & 17 & $49(1)$ & 17 & $100 \%$ \\
\hline 2 & 30 & $48(0)$ & 26 & $87 \%$ \\
\hline 3 & 27 & $44(13)$ & 22 & $81 \%$ \\
\hline 4 & 20 & $50(13)$ & 13 & $65 \%$ \\
\hline 5 & 38 & 50 ( 14) & 33 & $87 \%$ \\
\hline 6 & 20 & $50(13)$ & 14 & $70 \%$ \\
\hline 7 & 50 & $55(11)$ & 40 & $80 \%$ \\
\hline 8 & 25 & $50(4)$ & 20 & $80 \%$ \\
\hline 9 & 50 & $57(2)$ & 39 & $78 \%$ \\
\hline 10 & 25 & $47(2)$ & 21 & $84 \%$ \\
\hline 11 & 176 & 50 (18) & 139 & $79 \%$ \\
\hline 12 & 32 & $54(13)$ & 29 & $91 \%$ \\
\hline 13 & 50 & $49(13)$ & 43 & $86 \%$ \\
\hline 14 & 299 & $49(12)$ & 246 & $82 \%$ \\
\hline 15 & 35 & $48(8,2)$ & 48 & $100 \%$ \\
\hline 16 & 50 & $50(11)$ & 45 & $90 \%$ \\
\hline 17 & 50 & $47(11)$ & 46 & $92 \%$ \\
\hline 18 & 50 & $48(10)$ & 44 & $88 \%$ \\
\hline 19 & 30 & $44(8.9)$ & 27 & $90 \%$ \\
\hline 20 & 20 & $54(11)$ & 19 & $95 \%$ \\
\hline 21 & 25 & $48(10)$ & 36 & $90 \%$ \\
\hline 22 & 100 & 51 ( 11) & 92 & $92 \%$ \\
\hline 23 & 259 & $54(13)$ & 225 & $87 \%$ \\
\hline 24 & 69 & $49(6)$ & 64 & $96 \%$ \\
\hline 25 & 40 & $52(2)$ & 35 & $88 \%$ \\
\hline 26 & 71 & 47 (11) & 59 & $83 \%$ \\
\hline
\end{tabular}

saúde", sendo a média de pacientes incluídos de 179. Desta população, 30\% eram ativos no mercado de trabalho, 34\% inativos; $31 \%$ aposentados e $5 \%$ aposentados ativos.

A escolaridade foi avaliada em 5 teses, sendo 3 (60\%) sobre qualidade de vida e 2 (40\%) sobre economia de saúde. Correlacionando o nível de escolaridade com os períodos 
de estudo, verificou-se que esta análise começou a ser feita a partir do período III. Na década de 1990, nota-se um predomínio de pacientes com nível fundamental de ensino, seguidos de analfabetos. A partir de 2001, verifica-se um pequeno aumento de pacientes com nível médio e superior de ensino. Cabe ressaltar, no entanto, que, a partir deste período, os trabalhos iniciais em qualidade de vida excluíram pacientes analfabetos de seus estudos (Tabela 3 ).

TABELA 3

AVALIAÇÃo DESCRITIVA DO NÍVEL DE ESCOLARIDADE AO LONGO DOS ANOS OBSERVADOS

\begin{tabular}{cccccc}
\hline & analfabeto & $1^{\circ}$ grau & $2^{\circ}$ grau & $3^{\circ}$ grau & $\mathrm{n}$ \\
\hline 1993 & $8 \%$ & $92 \%$ & $0 \%$ & $0 \%$ & 25 \\
\hline 1997 & $18 \%$ & $82 \%$ & $0 \%$ & $0 \%$ & 50 \\
2001 & $0 \%$ & $82 \%$ & $16 \%$ & $2 \%$ & 50 \\
\hline $\begin{array}{c}1^{\circ} \text { tese } \\
2004\end{array}$ & $9 \%$ & $74 \%$ & $15 \%$ & $2 \%$ & 100 \\
\hline $\begin{array}{c}2^{\circ} \text { tese } \\
2004\end{array}$ & $13 \%$ & $75 \%$ & $11 \%$ & $3 \%$ & 259 \\
\hline
\end{tabular}

Obs.- Apenas 5 teses descreveram, de modo discriminado, a escolaridade.

Quanto à classificação funcional $(\mathrm{CF})$, verificou-se que, das 26 teses que fizeram esta avaliação, apenas $11(42,3 \%)$ incluíram as CF de I a IV em suas amostras, havendo um predomínio das CF I e II, conforme Figura 1.

Figura 1

DisTribuição DA CLASSIFICAÇÃO FUNCIONAL CONFORME OS PERÍODOS

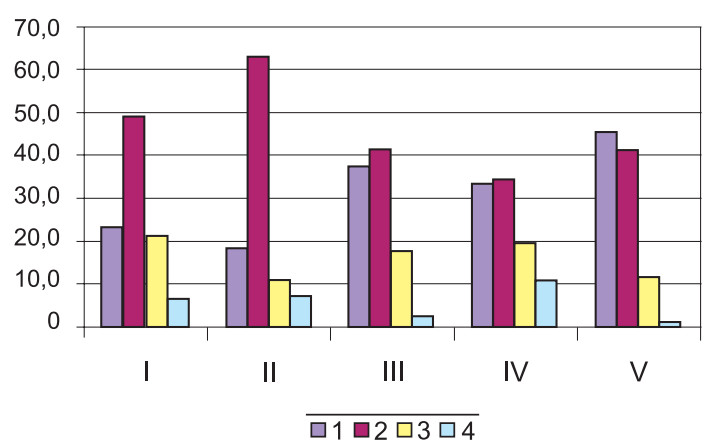

Figura 1 - I (1979- 1984); II (1985- 1989); III (1990-1994); IV (1995 - 1999); V (2000- 2004).

A Figura 2 apresenta as variações encontradas no perfil terapêutico utilizado pela população deste estudo,
Figura 2

AVALIAÇÃO DOS MEDICAMENTOS UTILIZADOS NAS TESES AO LONGO DE 25 ANOS

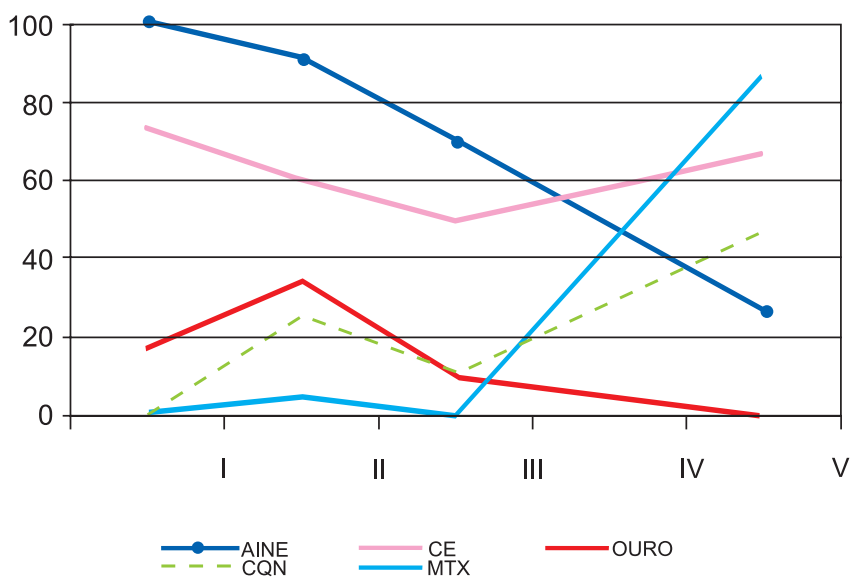

Figura 2 - AINE: antiinflamatórios; CE: corticosteróides; CQN: cloroquina/ hidroxicloroquina; MTX: metotrexato.

em correlação com cada período de tempo. No grupo I, evidenciou-se um predomínio do uso de antiinflamatórios (AINEs), seguido pelo uso de corticóides. Cabe ressaltar que, em ambos grupos I e II, observou-se uma freqüente associação entre classes de AINEs, o que se reduziu ao longo dos anos, mais substancialmente no último grupo. A análise do uso de corticóide apresentou uma variação peculiar. Inicialmente utilizado em larga escala, como evidenciado nos dois primeiros grupos, declinou substancialmente no grupo III e, posteriormente, observou-se, no último grupo, um aumento de sua utilização entre a população estudada. No que tange às drogas modificadoras de doença (DMARDs), especialmente ao metotrexato, verifica-se uma elevação linear na sua inserção como parte do arsenal terapêutico da AR. As teses pertencentes ao período IV não avaliaram tratamento. Nenhuma tese abordou associação entre DMARDs, nem medicamentos biológicos. A tabela 4 descreve os parâmetros clínicos e laboratoriais mais prevalentes entre as teses deste estudo, a saber: tempo de diagnóstico, HAQ e VHS. Quanto ao tempo de diagnóstico, verificamos uma discreta oscilação, com redução inicial no período II, voltando a se elevar nos períodos consecutivos. O valor do HAQ, no entanto, permanece elevado nos três períodos observados (Figura 5 ), em contradição ao declínio do VHS e à mudança do perfil terapêutico. Houve uma mudança nos tipos de estudo ao longo dos anos. Evidencia-se um aumento de temas versando sobre qualidade de vida, tal como o HAQ, passou a ser mais utilizado na prática assistencial. 
TABELA 4

AVALIAÇÃo DESCRITIVA DE PARÂMETROS CLÍNICOLABORATORIAIS MAIS ESTUDADOS NAS TESES

\begin{tabular}{cccc}
\hline $\begin{array}{c}\text { Período/ } \\
\text { parâmetro(média) }\end{array}$ & $\begin{array}{c}\text { Tempo de } \\
\text { diagnóstico } \\
\text { (anos) }\end{array}$ & $\begin{array}{c}\text { HAQ (Health } \\
\text { Assessment } \\
\text { Questionnaire) }\end{array}$ & VHS (mmHg) \\
\hline I: $1979-1984$ & 7.60 & Não disponivel & Não disponível \\
II: 1985 -1989 & 7.25 & Não disponível & 59 \\
III: $1990-1994$ & 8.60 & 1.143 & 25 \\
IV: $1995-1999$ & 8.54 & 0.94 & 39 \\
V: $2000-2004$ & 11 & 1.068 & 31 \\
\hline
\end{tabular}

VHS - Velocidade de hemossedimentação.

\section{DISCUSSÃO}

A ARé uma doença auto-imune, inflamatória, sistêmica, crônica e incapacitante, caracterizada pelo seu elevado impacto de morbidade e mortalidade ${ }^{(12)}$. As transformações experimentadas no conhecimento dos mecanismos imunológicos envolvidos na sua patogênese, no arsenal medicamentoso disponível e no paradigma do manejo clínico refletiram em uma avaliação rotineira mais racional e precisa, determinando uma assistência mais precoce e agressiva quanto ao uso dos imunossupressores em geral ${ }^{(13,14)}$. Na tentativa de analisar tais modificações, o estudo procurou avaliar o perfil clínico-laboratorial e sociodemográfico dos pacientes com AR que participaram de projetos de pós-graduação na EPM ao longo de 25 anos.

Os principais achados desta análise foram quanto à mudança flagrante observada nos tipos de estudo, perfil terapêutico e, em especial, quanto aos parâmetros de avaliação clínica e laboratorial dos pacientes com AR. A pesquisa clínico-qualitativa vem se solidificando nos últimos anos. Estudos sobre qualidade de vida se iniciaram na década de 90, ampliando-se substancialmente em seguida. Nos anos mais recentes, começam a apontar, também, estudos econômicos, talvez refletindo uma preocupação com o importante impacto sobre o sistema de saúde pública imposto pela doença ${ }^{(15)}$.

Os parâmetros clínicos acompanharam as modificações nos tipos de estudo observados. A análise de avaliação global de doença (AGD) pelo médico e pelo paciente; o número de articulações dolorosas (NAD) e o número de articulações edemaciadas (NAE) foram avaliados em um pequeno número de teses. O HAQ começou a ser utilizado a partir do grupo IV (1995 em diante). Seus valores elevados podem provavelmente refletir a natureza incapacitante da doença e a sua elevada morbidade. O tempo de diagnóstico, caracterizado pelo intervalo de tempo entre o início dos primeiros sintomas ao seu diagnóstico, além de ter permanecido constante, mostrou-se elevado. A relevância deste dado permite algumas hipóteses. Primeiro, que, a despeito de uma abordagem propedêutica e terapêutica mais agressivas, o diagnóstico ainda permanece tardio, talvez refletindo as dificuldades de acesso ao reumatologista no sistema público de saúde ${ }^{(16)}$. Segundo, como possível conseqüência da hipótese anterior, um diagnóstico protelado retardaria o início do tratamento, gerando seqüelas importantes, o que talvez implique na persistência de um HAQ elevado.

Paradoxalmente a esses achados, parâmetros de inflamação, representados pelo VHS, apresentaram um linear declínio ao longo dos anos, que pode sugerir um melhor controle da atividade de doença nos tempos atuais. Uma discreta mudança no nível de escolaridade também despertou a atenção. Embora ainda prevaleçam pacientes analfabetos e com nível fundamental de ensino, notou-se, nos anos mais recentes, uma tendência à participação de pacientes com nível de escolaridade mais elevado, incluindo aqueles com nível superior de ensino. Essa situação talvez reflita a perda do poder aquisitivo e a agregação do paciente até então com direito ao sistema privado e que hoje não têm mais condições de arcar com os custos elevados do mesmo. Outra possibilidade é o reconhecimento de uma assistência mais especializada em um serviço universitário e/ou piora da qualidade, no setor privado, na prestação de serviço de saúde especializado.

A relevância deste estudo se dá ao apontar possíveis questionamentos que nos permitam uma reflexão sobre algumas de suas características atuais, bem como as do sistema público de saúde. Critérios de diagnóstico bem definidos; incorporação tecnológica refinada, a utilização de técnicas laboratoriais e de imagem mais precisas que permitem maior precocidade do diagnóstico; e a utilização de opções terapêuticas disponíveis, conquanto elevem as expectativas de melhor manejo da doença, absorvem os já escassos recursos. Em contrapartida, ainda nos vemos, freqüentemente, diante de pacientes com seqüelas graves e mal informados sobre a sua doença. 


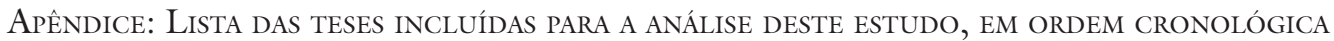

\begin{tabular}{|c|c|c|c|c|}
\hline Teses & Ano & Autor & Título da tese & Nivel \\
\hline 1 & 1979 & Maria Helena Torres Unzer & Avaliação funcional e histoimunopatológica do rim na artrite reumatóide & Doutor \\
\hline 2 & 1982 & José Goldenberg & Estudo de plaquetas em artrite reumatóide & Doutor \\
\hline 3 & 1987 & Maria de Fátima Lobato da Cunha & 0 músculo na artrite reumatóide: estudo anátomo-clínico-laboratorial & Mestre \\
\hline 4 & 1989 & Ângela Luiza Duarte Pinto Pessoa & $\begin{array}{l}\text { Estudo do fator reumatóide de pacientes com artrite reumatóide - capacidade de fixação do } \\
\text { complemento }\end{array}$ & Doutor \\
\hline 5 & 1990 & Marcos Bosi Ferraz & $\begin{array}{c}\text { Tradução para o português e validação do questionário para avaliar a capacidade funcional } \\
\text { 'Stanford Health Assessment Questionnaire' }\end{array}$ & Doutor \\
\hline 6 & 1992 & Henrique Josef & Estudo do idiotipo WA em pacientes com artrite reumatóide soropositiva & Doutor \\
\hline 7 & 1992 & José Tibúrcio de Monte Neto & Excreção de microproteínas urinárias e alterações renais na artrite reumatóide & Mestre \\
\hline 8 & 1993 & Marina Rodrigues Quaresma & Avaliação da corticoterapia na artrite reumatóide através do método utility & Mestre \\
\hline 9 & 1994 & Fábio Ikedo & Estudo prospectivo de alterações renais na artrite reumatóide & Mestre \\
\hline 10 & 1995 & Lenise Brandão & $\begin{array}{l}\text { Qualidade de vida em artrite reumatóide: validação de uma versão do Arthritis Impact } \\
\text { Measurement Scales } 2 \text { para a língua portuguesa (Brasil-AIMS2) }\end{array}$ & Mestre \\
\hline 11 & 1996 & Wilton Silva dos Santos & $\begin{array}{l}\text { Valor diagnóstico e significado clínico do fator antiperinuclear e anticorpo antiestratocórneo na } \\
\text { artrite reumatóide }\end{array}$ & Doutor \\
\hline 12 & 1996 & Leda Magalhães de Oliveira & $\begin{array}{l}\text { Evolução da capacidade funcional de pacientes com artrite reumatóide, avaliada pelo 'Stanford } \\
\text { Health Assessment Questionnaire' e escala EPM-ROM }\end{array}$ & Mestre \\
\hline 13 & 1997 & Rozana Mesquita Ciconelli & $\begin{array}{l}\text { Tradução para o português e validação do questionário genérico de avaliação de qualidade de } \\
\text { vida 'Medical Outcomes Study 36-Item Short-Form Health Survey (SF-36) }\end{array}$ & Doutor \\
\hline 14 & 1998 & Maria José Pereira Vilar & Estudo das alterações radiográficas das mãos e dos pés em pacientes com artrite reumatóide & Doutor \\
\hline 15 & 1999 & Maria Rosenilda Petronila de Carvalho & Gasto energético da caminhada em pacientes com artrite reumatóide & Mestre \\
\hline 16 & 1999 & Sandra Mara Meirelles & Avaliação isocinética do joelho de pacientes com artrite reumatóide & Mestre \\
\hline 17 & 1999 & Dircilene da Mota Falcão & $\begin{array}{l}\text { Processo de tradução e adaptação cultural de questionários de qualidade de vida: avaliação de } \\
\text { sua metodologia }\end{array}$ & Mestre \\
\hline 18 & 2001 & Adriana Bezerra d'Amorin & $\begin{array}{l}\text { Avaliação das formas auto-administradas dos questionários MHAQ e SF-12 em pacientes com } \\
\text { doenças reumatológicas }\end{array}$ & Mestre \\
\hline 19 & 2001 & Sandra Hiroko Watanabe & Avaliação isocinética do ombro reumatóide & Mestre \\
\hline 20 & 2002 & Paula Gabriel Silva. & $\begin{array}{l}\text { Avaliação da efetividade de uma órtese funcional para deformidade no polegar em botoeira tipo I e } \\
\text { II, na mão dominante em pacientes com artrite reumatóide: um estudo controlado e randomizado }\end{array}$ & Mestre \\
\hline 21 & 2003 & Adriana Garcia Orfale & $\begin{array}{c}\text { Tradução e validação do Disabilities of the arm, shoulder and hand (DASH) para a língua } \\
\text { portuguesa }\end{array}$ & Mestre \\
\hline 22 & 2004 & Gustavo da Cunha Chermont & Utilização de recursos e custos em artrite reumatóide & Mestre \\
\hline 23 & 2004 & Sérgio Cândido Kowalski & Valoração por contingência: validação do método em pacientes com artrite reumatóide & Doutor \\
\hline 24 & 2004 & Rita Nely Furtado & $\begin{array}{c}\text { Infiltração poliarticular com corticosteróide versus corticoterapia sistêmica em pacientes com } \\
\text { artrite reumatóide }\end{array}$ & Doutor \\
\hline 25 & 2004 & Wanda Chiyoko Iwakami Caldana & $\begin{array}{c}\text { A ressonância magnética do quadril na avaliação de pacientes com artrite reumatóide - estudo } \\
\text { descritivo }\end{array}$ & Mestre \\
\hline 26 & 2004 & Alberto Max Nieto Colônia & $\begin{array}{l}\text { Estudo longitudinal dos anticorpos fator reumatóide, antifator perinuclear e antipeptídeo } \\
\text { citrulinado cíclico e possíveis correlações com a evolução clínica e radiológica em uma coorte de } \\
\text { pacientes com artrite reumatóide }\end{array}$ & Doutor \\
\hline
\end{tabular}




\section{REFERÊNCIAS}

1. Case JP: Old and new drugs used in rheumatoid arthritis: a historical perspective. Part 1: the older drugs. Am J Ther 8: $23-43,2001$.

2. Kellgren JH, Jeffrey MR, Ball JF, editors. The epidemiology of chronic rheumatism. Vol. I. Philadelphia: FA Davis; 1963.

3. Moreland LW, Russell AS, Paulus HE: Management of rheumatoid arthritis: the historical context. J Rheumatol 28: 1431-52, 2001.

4. Mijiyawa M: Epidemiology and semiology of rheumatoid arthritis in Third World countries. Rev Rheum Engl Ed 62: 121-6, 1995.

5 Mottram PL: Past, present and future drug treatment for rheumatoid arthritis and systemic lupus erythematosus. Immunol Cell Biol 8: 350-3, 2003.

6. Graudal N: The natural history and prognosis of rheumatoid arthritis: association of radiographic outcome with process variables, joint motion and immune proteins. Scand J Rheumatol Suppl (118): 1-38, 2004.

7. Dooley MA; Hogan SL: Environmental epidemiology and risk factors for autoimmune disease. Curr Opin Rheumatol 15: 99 $103,2003$.

8. Hochberg MC, Chang RW, Dwosh I, Lindsey S, Pincus T, Wolfe F: The American College of Rheumatology 1991 revised criteria for the classification of global functional status in rheumatoid arthritis. Arthritis Rheum 35: 498-502, 1992.
9. Ferraz MB, Oliveira LM, Araújo PM, Atra E, Tugwell P: Crosscultural reliability of the physical ability dimension of the physical ability dimension of the health assessment questionnaire. J Rheumatol 17: 813-7, 1990.

10. Ropes MW, Bennett GA, Cobb S, Jacox R, Jessar RA: 1958 revision of diagnostic criteria for rheumatoid arthritis. Bull Rheum Dis 9: 175-6, 1958.

11. Arnett FC, Edworthy SM, Bloch DA, et al: The American Rheumatism Association 1987 revised criteria for the classification of rheumatoid arthritis. Arthritis Rheum 31: 315-24, 1988.

12. Cruz FA: Novo estudo sócio-econômico sobre as doenças reumáticas no Brasil. Rev Bras Reumatol 21: 114-8, 1981.

13. Bernelot Moens HJ, van de Laar MA, van der Korst JK: Comparison of the sensitivity and specificity of the 1958 and 1987 criteria for rheumatoid arthritis. J Rheumatol 19: 198-203, 1992.

14. Rantapaa-Dahlqvist S: Diagnostic and prognostic significance of autoantibodies in early rheumatoid arthritis. Scand J Rheumatol 34: 83-96, 2005.

15. Kremers HM, Nicola P, Crowson CS, O'Fallon WM, Gabriel SE: Therapeutic strategies in rheumatoid arthritis over a 40 -year period. J Rheumatol 31: 2366-73, 2004.

16. Muirden KD: Community Oriented Program for the Control of Rheumatic Diseases: studies of rheumatic diseases in the developing world. Curr Opin Rheumatol 17: 153-6, 2005. 